\title{
Incidental thyroid malignancies in patients operated for hyperthyroidism
}

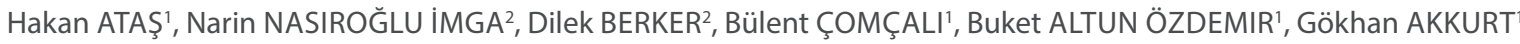 \\ ${ }^{1}$ Department of Breast and Endocrine Surgery, General Surgery Clinic, Ankara Numune Training and Research Hospital, Ankara, Turkey \\ ${ }^{2}$ Department of Endocrinology, Ankara Numune Research and Training Hospital, Ankara, Turkey \\ Correspondence \\ Hakan ATAŞ \\ Ankara Numune Eğitim ve Araştırma Hastanesi, Genel Cerrahi Kliniği, Meme ve Endokrin Cerrahisi Bölümü, Ankara, Türkiye \\ e-mail:drhakanatas@gmail.com
}

\begin{abstract}
This study aimed to investigate the frequency of incidental thyroid cancer in patients undergoing thyroidectomy due to different forms of hyperthyroidism and also explore the behavioral pattern of cancer in malignant cases.

A total of 1210 patients operated in the clinic between March 2014 and August 2018 were screened consecutively. Of these, 347 were thyrotoxic; $68.9 \%$ were female, and $31.1 \%$ were male. Further, $43.8 \%(n=152)$ had Graves' disease (GD), $4 \%(n=14)$ had toxic nodular goiter (TNG), and 52.2\% $(n=181)$ had toxic multinodular goiter (TMNG). Patients with hyperthyroidism were classified into two groups (Group 1: Benign; Group 2: Malignant). Data including age, sex, presence of GD, body mass index (BMI), thyroid volume, thyroid-stimulating hormone value at the time of diagnosis, TR-Ab, anti-TPO-Ab, and follow-up of hyperthyroidism were compared between the groups.

Thirty-eight (11\%) of all thyrotoxic patients had thyroid carcinoma. Sixteen (10.52\%) patients with GD, 2 (14.3\%) with TNG, and 20 (11\%) with TMNG had thyroid carcinoma. The histopathological examination revealed 35 papillary (21 microscopic), 1 follicular, and 2 cases of medullary thyroid carcinoma. The incidence of malignancy increased in patients with high BMI. Anti$\mathrm{TR}-\mathrm{Ab}$ and anti-TPO antibodies were found to be significantly higher in the malignant group.

The risk of developing thyroid cancer in different forms of hyperthyroidism is similar. Even in thyrotoxic patients, high BMI correlates with the development of thyroid cancer. It is believed that the cancer rate of $11 \%$ should not be underestimated. It is strongly recommended that toxic patients, especially patients with TNG, be closely monitored with USG and FNAC for possible malignancy development.
\end{abstract}

Key words: Graves' disease, hyperthyroidism, incidental thyroid cancer, thyroid carcinoma, thyrotoxicosis, toxic nodular goiter

\section{INTRODUCTION}

The incidence of thyroid cancer has increased in many countries in the last four decades, which might be attributed to the advances in diagnostic methods, an increase in pathological sampling, and, possibly, changes in the prevalence of risk factors (1). The most important etiological factor causing thyroid cancer is ionizing radiation, especially in childhood. Obesity, smoking, hypothyroidism, hormonal exposures, and some environmental pollutants are the other factors associated with thyroid cancer (1). However, the relationship between hyperthyroidism and thyroid cancer is still controversial. Hyperthyroidism is defined as hyperfunctioning of the thyroid gland; its prevalence in adults is approximately $1.2 \%$. Graves' disease (GD), toxic adenoma (TA), and toxic multinodular goiter (TMNG) are accepted as the most common causes (2). Until recently, hyperthyroidism was thought to be protective against the development of thyroid cancer due to the lower serum thyroid-stimulating hormone (TSH) concentration $(2,3)$. However, thyrotoxicosis did not protect against incidental papillary thyroid cancer (4). The coexistence of thyroid cancer and hyperthyroidism varied widely from $1.6 \%$ to $21.1 \%$ (5). It is difficult to explain this variability. Another point of discussion is whether hyperthyroidism-related thyroid cancers are more aggressive $(5,6)$. It is important to determine the incidence of malignancy in patients with hyperthyroidism and reveal the clinical behavioral pattern of incidental tumors. This is because the high incidence of malignancy may further emphasize the necessity of surgery among treatment options. Therefore, this study was conducted to evaluate the frequency of thyroid cancer in patients undergoing thyroidectomy due to different forms of hyperthyroidism and also examine the behavioral pattern of cancer in malignant cases. 


\section{MATERIALS AND METHODS}

The records of 1210 patients who underwent thyroid surgery for any reason at the Breast and Endocrine Surgery Unit of Ankara Immune Research and Training Hospital between March 2014 and August 2018 were screened consecutively. The institutional ethical committee approved the study (approval number: E-181981). Of the 1210 patients, 347 (28.6\%) were diagnosed with hyperthyroidism and included in the study. Hyperthyroidism was diagnosed by the presence of symptoms and signs of thyrotoxicosis with free triiodothyronine (FT3), free thyroxin (FT4), TSH, anti-TSH receptor antibody (TR-Ab), and anti-thyroperoxidase antibody (Anti-TPO-Ab) levels and detailed thyroid and neck ultrasonography before the surgery. The normal levels for thyroid autoantibodies in the laboratory were as follows: Tg-Ab, $<50 \mathrm{IU} / \mathrm{mL}$; TPO-Ab, $<10 \mathrm{IU} / \mathrm{mL}$; and TR-Ab $<9$ U/L (9-14 U/L borderline, >14 U/L positive). Patients with TSH levels lower than $0.35 \mu \mathrm{lU} / \mathrm{mL}$ and with normal FT3 and FT4 levels (subclinical) or abovenormal FT3 and FT4 levels (overt) $(>3.71 \mathrm{pg} / \mathrm{mL}$ and $>1.48 \mathrm{ng} / \mathrm{dL}$, respectively) were considered to have hyperthyroidism. Of these, patients with thyrotoxicosis accompanied by a diffuse goiter, an increased radioiodine uptake, and presence of high levels of circulating TR-Ab were diagnosed with GD.

Thyroidectomies with different indications such as Hashimoto's thyroiditis and histopathologically revealed thyroid cancer were excluded from the study. Patients who had a history of thyroid cancer in the family, patients with another diagnosed malignancy, and patients with abnormal results of fine-needle aspiration biopsy were also excluded. Another exclusion criterion was having a history of previous neck irradiation or RAI.

All patients with hyperthyroidism were treated with antithyroid drugs preoperatively; surgical treatment was performed after the patients became euthyroid. The surgical indications for patients with GD were patient preference $(52,34.2 \%)$, ophthalmopathy $(12$, $7.8 \%$ ), inefficacy of antithyroid drug treatment, serious side effects such as hepatotoxicity and agranulocytosis $(79,51.9 \%)$, and compressive symptoms caused by a large volume of thyroid $(9,5.9 \%)$. The preferred surgical method was total thyroidectomy for almost all patients except patients with TNG. Nine of 14 patients with TNG were treated with lobectomy; the remaining 5 patients underwent subtotal thyroidectomy because of compression symptoms. Patients with TMNG also underwent total thyroidectomy with the indica- tions of compression, patient preference, and having a nodule larger than $4 \mathrm{~cm}$.

After the final examination of collected data and histopathological results, patients with hyperthyroidism were classified into two groups (Group 1: Benign; Group 2: Malignant). Data including age, sex, presence of $\mathrm{GD}$, body mass index (BMI), thyroid volume (TV), TSH value at the time of diagnosis, TR-Ab, anti-TPO$A b$, and follow-up of hyperthyroidism were compared between the two groups.

The statistical analysis was performed using SPSS version 21 software. The eligibility of numerical variables to normal distribution was investigated using Kolmogorov-Smirnov or Shapiro-Wilk tests. Descriptive analyses were performed using the mean \pm standard deviation for normally distributed numerical variables and the median and interquartile range for variables with no normal distribution. Normally distributed parameters were compared using the Student $t$ test, and the parameters not showing normal distribution were compared using the Mann-Whitney $\mathrm{U}$ test. The differences between these groups were compared using the chi-square or Fisher tests. Correlation coefficients and statistical significance were calculated using Spearman's test for evaluating the correlation between variables. In the multivariate analysis, the possible factors determined in univariate analyses were evaluated using logistic regression. A $P$ value less than 0.05 was considered statistically significant.

\section{RESULTS}

Of the 1210 patients treated surgically, 347 were operated for thyrotoxicosis. Of these 347 patients, 239 (68.9\%) were female, and 108 (31.1\%) were male; the mean age of the whole group was $48.27 \pm 13.31$ (34-63) years. Among these patients, 152 (43.8\%) had GD, 14 (4\%) had TNG, and 181 (52.2\%) had TMNG. The patient characteristics and clinicopathological features associated with malignancy in patients with hyperthyroidism are shown in Table 1. No significant difference was observed between groups in terms of age, sex, presence of GD, TV, serum TSH levels, and follow-up time of hyperthyroidism. However, the incidence of malignancy increased in patients with high BMI. Also, the levels of anti-TR-Ab and anti-TPO antibodies were found to be significantly higher in the malignant group.

Thyroid carcinoma was found in $11 \%(n=38)$ of all thyrotoxic patients. Sixteen (24.7\%) patients with 
Table 1: Patient characteristics and clinicopathologic features associated with malignancy in patients with hyperthyroidism.

\begin{tabular}{|c|c|c|c|}
\hline Characteristic & $\begin{array}{l}\text { Group 1/Benign }(n=309) \\
(n, \%)^{*}\end{array}$ & $\begin{array}{l}\text { Group 2/Malignant }(n=38) \\
(n, \%)^{*}\end{array}$ & $P$ value \\
\hline Age (year) & $47.8 \pm 13.5$ & $52.1 \pm 11.6$ & 0.056 \\
\hline \multicolumn{4}{|l|}{ Sex } \\
\hline Female & $213(89.1)$ & $26(10.9)$ & \multirow{2}{*}{0.949} \\
\hline Male & $96(88.9)$ & $12(11.1)$ & \\
\hline \multicolumn{4}{|l|}{ Presence of GD } \\
\hline Yes & $136(44.0)$ & $16(42.1)$ & \multirow{2}{*}{0.823} \\
\hline No & $173(56.0)$ & $22(57.9)$ & \\
\hline GD & $136(89.5)$ & $16(10.5)$ & \multirow{3}{*}{0.527} \\
\hline TNG & $12(85.7)$ & $2(14.3)$ & \\
\hline TMNG & $161(89)$ & $20(11)$ & \\
\hline BMI $\left(\mathrm{kg} / \mathrm{m}^{2}\right)$ & $26.6 \pm 3.9$ & $29.3 \pm 4.8$ & $<0.001$ \\
\hline TV $(m L)$ & $73.3 \pm 67.5$ & $86.1 \pm 73.6$ & 0.281 \\
\hline $\mathrm{TSH}(\mu \mathrm{IU} / \mathrm{mL})$ & $0.06 \pm 0.05$ & $0.06 \pm 0.03$ & 0.786 \\
\hline TR-Ab (U/L) & $18.1 \pm 33.5$ & $42.9 \pm 81.2$ & 0.039 \\
\hline Anti-TPO-Ab (IU/mL) & $122.6 \pm 262.3$ & $15.1 \pm 22.2$ & 0.049 \\
\hline $\begin{array}{l}\text { Follow-up of hyperthyroidism } \\
\text { (months) }\end{array}$ & $22 \pm 3$ & $26 \pm 8$ & 0,590 \\
\hline
\end{tabular}

anti-Tg-Ab: Anti-thyroglobulin antibody anti-TPO: Anti-thyroperoxidase antibody BMI: Body mass index GD: Graves' disease Tg: Thyroglobulin TMNG: Toxic multinodular goiter TNG: Toxic nodular goiter TR-Ab: Anti-TSH receptor antibody TSH: Thyroid-stimulating hormone TV: Thyroid volume. "Mean \pm standard deviation values represented for continuous variables.

GD, 2 (14.3\%) with TNG, and 20 (11\%) with TMNG had thyroid carcinoma. Papillary thyroid microcarcinoma (PTMC) was defined as a papillary thyroid carcinoma less than $10 \mathrm{~mm}$ in size. The histological examination revealed 21 (6.1\%) micropapillary, 14 (4.0\%) papillary, $1(0.3 \%)$ follicular carcinoma, and $2(0.6 \%)$ medullary thyroid carcinoma with a median tumor size of 10 $\mathrm{mm}$. Pathological diagnoses in different subgroups of patients with hyperthyroidism are summarized in Table 2. No statistically significant difference in the cancer rate was found between the different forms of hyperthyroidism.

The carcinomas were multifocal (MF) in eight patients (two had GD and six had TMNG; unilateral in three patients and bilateral in five patients). Thyroid capsule invasion $(\mathrm{TCl}$ ) and extra-thyroidal invasion (ETI) were found in seven (three with $G D$, one with TNG, and three with TMNG) and four patients (one with GD and three with TMNG), respectively. Neurovascular invasion (NVI) was found in only one patient with TMNG.
The pathological examination revealed lymph node invasions in the central compartment not detected by preliminary ultrasound in five patients (two with GD and three with TMNG). One of these patients had metastasis in the lateral compartment (right level 3) 14 months after total thyroidectomy.

The mean duration of the follow-up until the time of surgery for the benign and malignant groups was $22 \pm 3$ and $26 \pm 8$ months, respectively. All patients were alive and free of disease at the time of follow-up after the surgery.

\section{DISCUSSION}

MIn the present study, incidental thyroid carcinoma was histopathologically revealed in $11 \%$ of surgically treated patients due to hyperthyroidism. However, previous studies reported a wide range of the incidence of thyroid cancer in thyrotoxic patients. This wide range can be explained by geographic changes, iodine deficiency, radiation exposure, differences in surgical indication criteria, and differences in the extent of resection 
Table 2: Patient characteristics and clinicopathologic features associated with malignancy in patients with hyperthyroidism.

\begin{tabular}{lccccc}
\hline & $\begin{array}{c}\text { All patient }(\mathrm{n}=347) \\
(\mathrm{n}, \%)^{*}\end{array}$ & $\begin{array}{c}\text { GD }(\mathrm{n}=152) \\
(\mathrm{n}, \%)^{*}\end{array}$ & $\begin{array}{c}\text { TNG }(\mathrm{n}=14) \\
(\mathrm{n}, \%)^{*}\end{array}$ & $\begin{array}{c}\text { TMNG }(\mathrm{n}=181) \\
(\mathrm{n}, \%)^{*}\end{array}$ & P value \\
\hline Total number of malignancies & $38,11.0$ & $16,10.5$ & $2,14.3$ & $20,11.0$ & $\mathrm{NS}$ \\
PTMC & $21,6.0$ & $8,5.2$ & $1,7.1$ & $12,6.6$ & $\mathrm{~N}$ \\
PTC & $14,4.0$ & $8,5.2$ & - & -30.0 & 0.018 \\
FTC & $1,0.2$ & - & $1,0.5$ & NS \\
MTC & $2,0.5$ & - & $1,7.1$ & 0.5 & NS \\
TS $(\mathrm{mm})$ & $10(3-22)$ & $7(3-13.5)$ & $8.5(6-11)$ & $7(4-22)$ & NS \\
\hline
\end{tabular}

FTC: Follicular thyroid carcinoma GD: Graves' disease MTC: Medullary thyroid carcinoma NS: Nonsignificant PTC: Papillary thyroid carcinoma PTMC: Papillary thyroid microcarcinoma TMNG: Toxic multinodular goiter TNG: Toxic nodular goiter TS: Tumor size.

*Median (min-max) values are represented for continuous variables.

as well as in the number of histological sections examined per specimen. The prevalence of thyroid cancer in different forms of hyperthyroidism has been reported as $0 \%-16.6 \%$ in patients with $G D, 2.5 \%-12 \%$ in patients with TA, and $1.6 \%-8.2 \%$ in patients with TMNG (7-9). In the present study, the malignancy rate was $10.52 \%$, $14.3 \%$, and $11 \%$, respectively in patients with GD, TA, and TMNG, respectively. These rates were slightly higher than the rates in previous studies. The rate reported in another study was $7 \%, 6 \%$, and $8 \%$, respectively; thyroid carcinoma was found in $7.5 \%$ of patients with hyperthyroidism (7). The iodine deficiency in Turkey cannot account for this increase over the years. Further, the iodine prophylaxis program launched in 1999 did not consider this iodine deficiency as very severe. Another explanation for this increased risk of thyroid cancer following the diagnosis of hyperthyroidism could be TSH mimicking the effects of thyroid-stimulating antibody (TSAb), which might support the role of proto-oncogenes such as RET and TRK in the development of papillary thyroid cancer (10). Hyperthyroidism is associated with changes in the levels of sex steroid-binding globulin and sex steroid hormones, including elevations in estradiol levels, which is known to have a direct effect on thyroid carcinogenesis (10-12).

Table 1 shows that the serum TR-Ab levels were significantly higher in the malignant group.

This was probably because of the interaction of the circulating TR-Ab with TSH receptors on tumors or metastatic thyroid tissues, where it acted as a stimulant for thyrocyte growth $(6,13)$. The association between $\mathrm{BMI}$ and thyroid cancer was another significant finding of the present study. The results demon- strated that higher BMI was significantly associated with the increased risk of thyroid cancer, as shown by Son et al. (14); however, many studies showed no significant relationship (15). In the present study, this relationship was evaluated in the thyrotoxic patient group, unlike other studies.

In this study, three different types of thyroid cancer were shown to be associated with hyperthyroidism. The most common histological type of thyroid cancer associated with hyperthyroidism is papillary cancer, followed by follicular, medullary, and anaplastic cancers. In this study, the medullary type was the second most common type, with no anaplastic cancer found $(5,16)$. Although Cappelli et al. $(6)$ showed that thyroid cancer was more common in patients with GD compared with TNG and TMNG, the rate of malignancy was higher in patients with TNG and TMNG in the present study. Similar results have also been reported by Senyurek Giles et al. (17). The 2015 guidelines of the American Thyroid Association stated that hyperfunctioning nodules rarely showed malignant transformation (18). However, the rate of malignancy was the highest in patients with TNG in the present study (Table 2). Therefore, it should be kept in mind that a preoperative cytological examination is beneficial in patients with TNG.

In previous studies, the aggressiveness of thyroid cancers associated with hyperthyroidism increased in patients with $\operatorname{GD}(6,19,20)$. As mentioned earlier in the Results section, an evaluation was performed to reveal the behavioral pattern of cancer by means of the histopathological outcomes of patients with coexistent hyperthyroidism and thyroid cancer. Despite 
no statistically significant difference between tumor sizes in the three groups, small sample size failed to provide a statistically accurate assessment of multifocality, $\mathrm{TCl}, \mathrm{ETI}, \mathrm{NVI}$, and lymph node involvement in this study. Therefore, it was not possible to comment on which subgroups of hyperthyroidism-associated cancers were more aggressive.

The most important limitations of this study were small sample size and retrospective nature. To find out the actual incidence of thyroid cancer in hyperthyroid patients, it is necessary to operate all patients diagnosed with hyperthyroidism and include all patients suspected of having nodules in this group. However, such a prospective study design is not easy. Nevertheless, prospective studies with larger series may be useful, especially in determining the true incidence and etiopathogenesis of thyroid cancer in thyrotoxic patients.

In summary, this study demonstrated that the risk of developing thyroid cancer in different forms of hyperthyroidism was similar, and high BMI correlated with the development of thyroid cancer even in thyrotoxic patients. Since hyperthyroidism is a benign disease, the treatment approach in some patients with GD is the use of antithyroid drugs. Patients with TNG or TMNG are also treated with thyroid ablation after diagnosis; sometimes, they receive long-term antithyroid treatments. This often leads to a disregard of the risk of thyroid cancer and delay in the decision of thyroidectomy. It is believed that the cancer rate of $11 \%$ should not be underestimated. It is strongly recommended that toxic patients, especially patients with TNG, be closely monitored with USG and FNAC for possible malignancy development.

Conflicts of Interest: The authors declare no conflicts of interest.

Financial Disclosure: None declared.

Ethical approval: The local ethics committee approval was received for this study.

Informed consent: Informed consent was obtained from all individual participants in the study.

\section{REFERENCES}

1. Bray F, Ferlay J, Soerjomataram I, Siegel RL, Torre LA and Jemal A. Global cancer statistics 2018: GLOBOCAN estimates of incidence and mortality worldwide for 36 cancers in 185 countries. CA: a cancer journal for clinicians 2018; 68(6), 394-424.

2. Haymart MR, Repplinger DJ, Leverson GE, Elson DF, Sippel RS, Jaume JC \& Chen $\mathrm{H}$. Higher serum thyroid stimulating hormone level in thyroid nodule patients is associated with greater risks of differentiated thyroid cancer and advanced tumor stage. The Journal of Clinical Endocrinology and Metabolism 2008; 93(3), 809-814.
3. Fiore E, Vitti P. Serum TSH and risk of papillary thyroid cancer in nodular thyroid disease. The Journal of Clinical Endocrinology \& Metabolism 2012; 97(4), 1134-1145.

4. Preece J, Grodski S, Yeung M, Bailey M, Serpell J. Thyrotoxicosis does not protect against incidental papillary thyroid cancer. Surgery 2014; 156(5), 1153-1156.

5. Pazaitou-Panayiotou K, Michalakis K, Paschke R. Thyroid cancer in patients with hyperthyroidism. Hormone and metabolic research 2012; 44(04), 255-262.

6. Cappelli C, Braga M, De Martino E, Castellano M, Gandossi E, Agosti B, Rosei EA. Outcome of patients surgically treated for various forms of hyperthyroidism with differentiated thyroid cancer: experience at an endocrine center in Italy. Surgery today 2006; 36(2), 125-130.

7. Berker D, Isik S, Ozuguz U, Tutuncu YA, Kucukler K, Akbaba G, Guler S. Prevalence of incidental thyroid cancer and its ultrasonographic features in subcentimeter thyroid nodules of patients with hyperthyroidism. Endocrine 2011; 39(1), 13-20.

8. Angusti T, Codegone A, Pellerito R, Favero A. Thyroid cancer prevalence after radioiodine treatment of hyperthyroidism. The Journal of Nuclear Medicine 2000; 41(6), 1006.

9. Pacini F, Elisei R, Di Coscio GC, Anelli S, Macchia E, Concetti R, Pinchera A. Thyroid carcinoma in thyrotoxic patients treated by surgery. Journal of endocrinological investigation 1988; 11(2), 107-112.

10. Liang L, Zheng XC, Hu MJ, Zhang Q, Wang SY, Huang F. Association of benign thyroid diseases with thyroid cancer risk: a meta-analysis of prospective observational studies. Journal of endocrinological investigation 2019; 42(6), 673-685.

11. Krassas GE, Poppe K, Glinoer D. Thyroid function and human reproductive health. Endocrine reviews 2010; 31(5), 702-755.

12. Kumar A, Klinge CM, Goldstein RE. Estradiol-induced proliferation of papillary and follicular thyroid cancer cells is mediated by estrogen receptors $\alpha$ and $\beta$. International journal of oncology 2010; 36(5), 1067-1080.

13. Filetti $S$, Belfiore A, Amir SM, Daniels GH, Ippolito O, Vigneri $R$ Ingbar SH. The role of thyroid-stimulating antibodies of Graves' disease in differentiated thyroid cancer. New England Journal of Medicine 1988; 318(12), 753-759.

14. Son $\mathrm{H}$, Lee $\mathrm{H}$, Kang $\mathrm{K}$, Lee $\mathrm{I}$. The risk of thyroid cancer and obesity: a nationwide population-based study using the Korea National Health Insurance Corporation cohort database. Surgical oncology 2018; 27(2), 166-171.

15. Stansifer KJ, Guynan JF, Wachal BM, Smith RB. Modifiable risk factors and thyroid cancer. Otolaryngology-Head and Neck Surgery 2015; 152(3), 432-437.

16. Sharma SD, Kumar G, Guner K, Kaddour H. Hyperthyroidism in patients with thyroid cancer. Ear, Nose \& Throat Journal 2016; 95(6), 236-239.

17. Giles Y, Fatih T, Harika B, Yersu K, Tarik T, Serdar T. The risk factors for malignancy in surgically treated patients for Graves' disease toxic multinodular goiter, and toxic adenoma. Surgery 2008; 144(6), 1028-1037.

18. Haugen BR, Alexander EK, Bible KC, Doherty GM, Mandel SJ, Nikiforov YE, Schuff KG. 2015 American Thyroid Association management guidelines for adult patients with thyroid nodules and differentiated thyroid cancer: the American Thyroid Association guidelines task force on thyroid nodules and differentiated thyroid cancer. Thyroid 2016; 26(1), 1-133.

19. Belfiore A, Garofalo MR, Giuffrida D, Runello F, Filetti $S$, Fiumara A, Vigneri R. Increased aggressiveness of thyroid cancer in patients with Graves' disease. The Journal of Clinical Endocrinology \& Metabolism 1990; 70(4), 830-835.

20. Pellegriti G, Belfiore A, Giuffrida D, Lupo L, Vigneri R. Outcome of differentiated thyroid cancer in Graves' patients. The Journal of Clinical Endocrinology \& Metabolism 1998; 83(8), 28052809. 